The Astrophysical Journal, 601:1013-1018, 2004 February 1

(C) 2004. The American Astronomical Society. All rights reserved. Printed in U.S.A.

\title{
THE EARLY OPTICAL AFTERGLOW OF GRB 030418 AND PROGENITOR MASS LOSS
}

\author{
E. S. Rykoff, ${ }^{1}$ D. A. Smith, ${ }^{1}$ P. A. Price, ${ }^{2}$ C. W. Akerlof, ${ }^{1}$ M. C. B. Ashley, ${ }^{3}$ D. Bizyaev,,${ }^{4,5}$ G. J. Garradd, ${ }^{6}$ T. A. McKay, \\ R. H. McNaught, ${ }^{6}$ A. Phillips, ${ }^{3}$ R. Quimby, ${ }^{7}$ B. Schaefer,${ }^{8}$ B. Schmidt, ${ }^{6}$ W. T. Vestrand, ${ }^{9}$ J. C. Wheeler, ${ }^{7}$ And J. Wren ${ }^{9}$ \\ Received 2003 August 19; accepted 2003 October 16
}

\begin{abstract}
The ROTSE-IIIa telescope and the SSO 40 inch $(1.0 \mathrm{~m})$ telescope, both located at Siding Spring Observatory, imaged the early-time afterglow of GRB 030418. In this report, we present observations of the early afterglow, first detected by the ROTSE-IIIa telescope $211 \mathrm{~s}$ after the start of the burst and only $76 \mathrm{~s}$ after the end of the gamma-ray activity. We detect optical emission that rises for $\sim 600 \mathrm{~s}$, slowly varies around $R=17.3$ mag for $\sim 1400 \mathrm{~s}$, and then fades as a power law of index $\alpha=-1.36$. Additionally, the ROTSE-IIIb telescope, located at McDonald Observatory, imaged the early-time afterglow of GRB 030723 . The behavior of this light curve was qualitatively similar to that of GRB 030418, but 2 mag dimmer. These two afterglows are dissimilar to other afterglows such as GRB 990123 and GRB 021211. We investigate whether or not the early afterglow can be attributed to a synchrotron break in a cooling synchrotron spectrum as it passes through the optical band, but we find that this model is unable to accurately describe the early light curve. We present a simple model for gammaray burst emission emerging from a wind medium surrounding a massive progenitor star. This model provides an effective description of the data and suggests that the rise of the afterglow can be ascribed to extinction in the local circumburst environment. In this interpretation, these events provide further evidence of the connection between gamma-ray bursts and the collapse of massive stars.
\end{abstract}

Subject heading: gamma rays: bursts

\section{INTRODUCTION}

Around half of all well-localized gamma-ray bursts (GRBs) have resulted in the detection of optical counterparts. This low success rate is partly due to the difficulty in obtaining prompt coordinates, and so it has been argued that many GRB afterglows fade too rapidly for discovery by late-time follow-up observations. It is also possible that extinction from dense circumburst environments may cut optical emission below detectable levels (Klose et al. 2003). GRB 990123 remains unique as the only burst from which prompt optical emission was detected during gamma-ray emission (Akerlof et al. 1999), despite much effort from small rapidly responding telescopes such as ROTSE-I (Robotic Optical Transient Search Experiment I) and LOTIS (Livermore Optical Transient Imaging

\footnotetext{
12477 Randall Laboratory, University of Michigan at Ann Arbor, 500 East University Avenue, Ann Arbor, MI 48104-1120; erykoff@umich.edu, donaldas@umich.edu, akerlof@umich.edu, tamckay@umich.edu.

${ }^{2}$ Institute for Astronomy, University of Hawaii, 2680 Woodlawn Drive, Honolulu, HI 96822; price@ifa.hawaii.edu.

${ }^{3}$ School of Physics, Department of Astrophysics and Optics, University of New South Wales, Sydney, NSW 2052, Australia; mcba@phys.unsw.edu.au, a.phillips@unsw.edu.au.

${ }^{4}$ Department of Physics, University of Texas at El Paso, El Paso, TX 79968; dmbiz@baade.physics.utep.edu.

${ }^{5}$ Sternberg Astronomical Institute, MV Lomonosov Moscow State University, Universitetsky Pr. 13, 119899 Moscow, Russia.

${ }^{6}$ Research School of Astronomy and Astrophysics, Mount Stromlo Observatory, Cotter Road, Weston Creek, Canberra, ACT 2611, Australia gloomberah@ozemail.com.au, rmn@murky.anu.edu.au, brian@mso.anu. edu.au.

Department of Astronomy, University of Texas at Austin, RLM 15.308, C-1400, Austin, TX 78712-1083; quimby@astro.as.utexas.edu,wheel@astro. as.utexas.edu.

${ }^{8}$ Department of Physics and Astronomy, Louisiana State University, Baton Rouge, LA 70803; brad@baton.phys.lsu.edu.

9 Los Alamos National Laboratory, NIS-2, MS D436, Los Alamos, NM 87545; vestrand@lanl.gov, jwren@nis.lanl.gov.
}

System; Akerlof et al. 2000; Kehoe et al. 2001; Park et al. 1999). Larger fast-slewing telescopes such as ROTSE-IIIa have since come online in an effort to achieve deeper imaging at early times. To date, only two other afterglows have been detected within 10 minutes of the burst - GRB 021004 (Fox et al. 2003c) and GRB 021211 (Fox et al. 2003b; Li et al. 2003) - and both of these were detected only after the afterglow began to decay.

In this paper, we report on early-time optical observations of GRB 030418 with the ROTSE-IIIa telescope and the SSO 40 inch $(1.0 \mathrm{~m})$ telescope, both located at Siding Spring Observatory, Australia. We also report on early-time optical observations of GRB 030723 with the ROTSE-IIIb telescope at McDonald Observatory, Texas. Despite rapid responses to each of these bursts ( 211 and $47 \mathrm{~s}$ respectively), we have no evidence of prompt optical counterparts. We present here a physical model that ascribes the afterglow rise to extinction in the local circumburst environment.

The ROTSE-III array is a worldwide network of $0.45 \mathrm{~m}$ robotic, automated telescopes, built for fast $(\sim 6 \mathrm{~s})$ responses to GRB triggers from satellites such as HETE-2 (High Energy Transient Explorer 2$)$. They have a wide $(1.85 \times 1.85)$ field of view imaged onto a Marconi $2048 \times 2048$ back-illuminated thinned CCD and operate without filters. The ROTSE-III systems are described in detail in Akerlof et al. (2003).

The SSO 40 inch telescope has an $\mathrm{f} / 8$ direct imager at a Cassegrain focus. The field of view has a 20 ' 8 diameter on a Tek $2048 \times 2048 \mathrm{CCD}$ with $24 \mu \mathrm{m}$ pixels. The telescope can be operated unfiltered or with a range of filters. For these observations, the $\mathrm{CCD}$ was used with $2 \times 2$ binning giving pixels of 1.22 .

\section{OBSERVATIONS AND ANALYSIS}

On 2003 April 18, HETE-2 detected GRB 030418 (HETE-2 trigger 2686) at 9:59:18.85 UT. The first determination of its 
position was distributed as a GRB Coordinates Network (GCN) notice at 10:02:54 UT, with a $28^{\prime}$ radius error box, $205 \mathrm{~s}$ after the start of the burst (HETE-2 2686; sequence 2). Ground analysis improved the error radius to $18^{\prime}$, and a second GCN notice was distributed at 11:43:01 UT (HETE-2 2686; 4). The burst was determined to have lasted $135 \mathrm{~s}$, with fluences of $1.2 \times 10^{-6} \mathrm{ergs} \mathrm{cm}^{-2}(2-25 \mathrm{keV})$ and $2.5 \times 10^{-6} \mathrm{ergs} \mathrm{cm}^{-2}$ (30-400 keV), classifying it as a long, X-ray-rich GRB (Shirasaki et al. 2003). The burst occurred while the Moon was bright (96\% illumination), and very few telescopes reported follow-up observations.

ROTSE-IIIa responded automatically to the first GCN notice in under $6 \mathrm{~s}$ with the first exposure starting at 10:03:00 UT, $211 \mathrm{~s}$ after the burst and only $76 \mathrm{~s}$ after the cessation of gamma-ray activity. The automated scheduling software began a program of $105 \mathrm{~s}$ exposures followed by $9020 \mathrm{~s}$ exposures. Longer exposures were not taken because the bright Moon would have saturated the images. The second GCN notice triggered ROTSE-IIIa to repeat the same sequence of observations. Analysis of the individual frames in near real time did not reveal any new source brighter than the unfiltered limiting magnitude of $\sim 16$.

After the receipt of the second GCN notice, Price et al. (2003) initiated a burst response on the SS0 40 inch telescope, beginning $7139 \mathrm{~s}$ after the burst, with an unfiltered $100 \mathrm{~s}$ image, followed by a sequence of $10300 \mathrm{~s}$ images taken with a Johnson $R$-band filter. Price et al. (2003) identified a new object at $\alpha=10^{\mathrm{h}} 54^{\mathrm{m}} 33^{\mathrm{s}} .674, \delta=-7^{\circ} 01^{\prime} 40^{\prime \prime} .75$ (J2000.0) at $\sim 18.8$ mag that was not on the Digitized Sky Survey red plates (Price et al. 2003).

Co-adding sets of 10 ROTSE-IIIa images revealed the optical counterpart reported by Price et al. (2003). As can be seen in Figure 1, the afterglow is barely detected in the first ROTSE-IIIa image, while a nearby 18 th magnitude star at $\alpha=10^{\mathrm{h}} 54^{\mathrm{m}} 32^{\mathrm{s}} .6, \delta=-07^{\circ} 03^{\prime} 38^{\prime \prime} \cdot 0$ (J2000.0) is clearly visible. The afterglow then increases in brightness, exceeding the comparison star, before fading below our detection threshold. Using an identical analysis as that for the afterglow itself (described below), we measured the fluctuations of the comparison star to be less than $0.1 \mathrm{mag}$. The comparison star is detected with a signal-to-noise ratio $(\mathrm{S} / \mathrm{N})$ of 7.7 in the first images and a $\mathrm{S} / \mathrm{N}$ of greater than 10 in subsequent images.

The ROTSE-IIIa data and the first SSO 40 inch observation were taken without filters. Further SSO 40 inch observations were taken with an $R$-band filter. Therefore, it is important to bring both sets of measurements to the same standard photometric system. We compared each image with the standard $R$-band photometric calibration from the USNO $1 \mathrm{~m}$ telescope

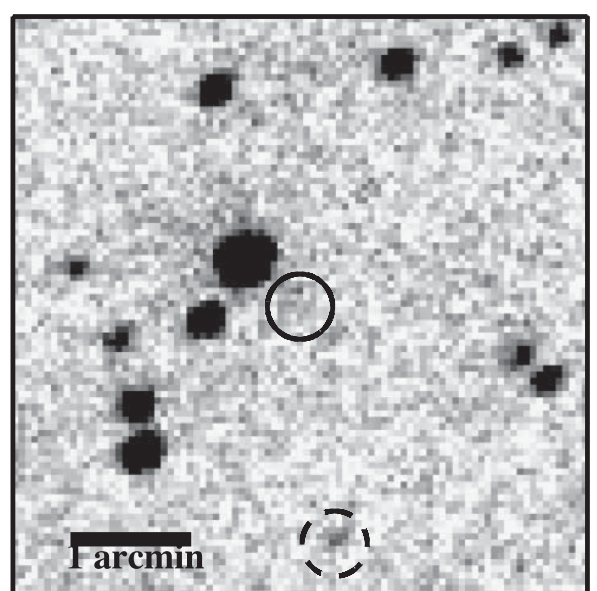

Start $=221.1$ sec End $=355.0 \mathrm{sec}$

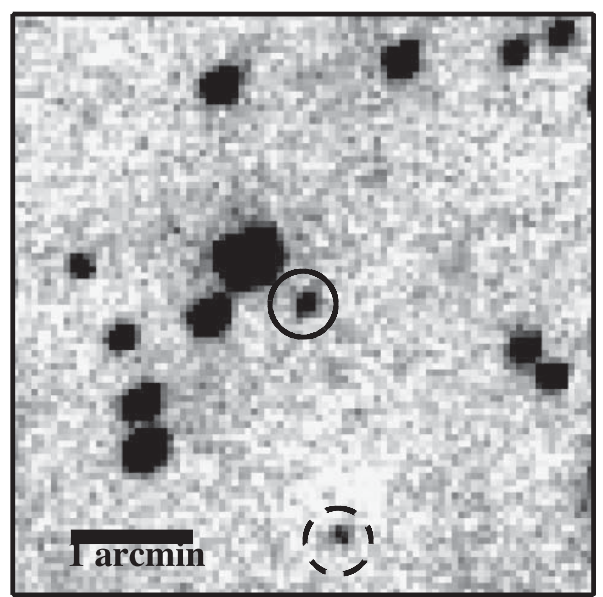

Start $=656.1 \mathrm{sec}$ End $=940.6 \mathrm{sec}$

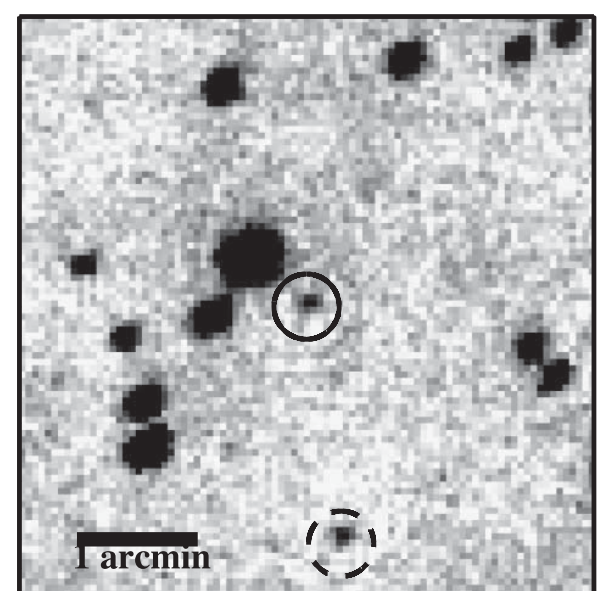

Start $=364.2 \mathrm{sec}$ End $=\mathbf{6 4 6 . 8 \mathrm { sec }}$

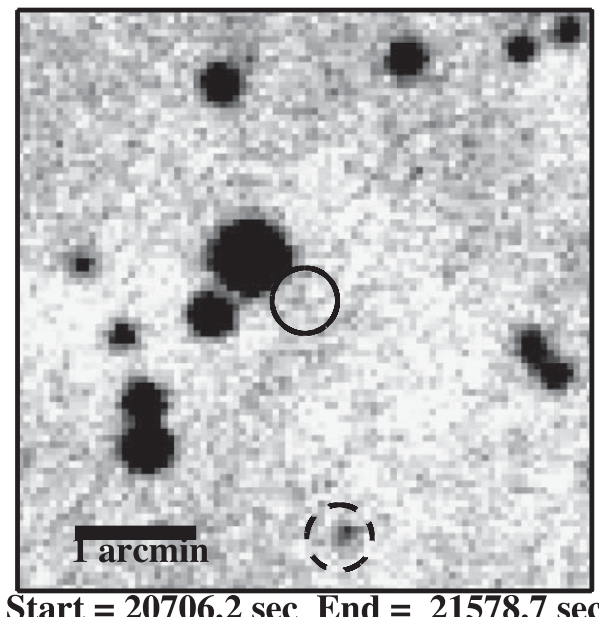

FIG. 1.-Mosaic of co-added images taken by ROTSE-IIIa starting $211 \mathrm{~s}$ after the burst that clearly show the rise and fall of the afterglow of GRB 030418 . In the first image, the 18th magnitude comparison star (dashed circle) is clearly visible, while the afterglow is not. In subsequent images, the afterglow (solid circle) gets brighter than the comparison star and fades away. The four images have different total exposure times, and the comparison star varies by $<0.1$ mag. 
TABLE 1

Optical Photometry for GRB 030418

\begin{tabular}{|c|c|c|c|c|}
\hline Telescope & Filter & $\begin{array}{l}\text { Start } \\
\text { (s) }\end{array}$ & $\begin{array}{c}\text { End } \\
\text { (s) }\end{array}$ & Magnitude \\
\hline \multirow[t]{12}{*}{ ROTSE-IIIa ......................... } & None & 221.1 & 355.0 & $18.76 \pm 0.35$ \\
\hline & None & 364.2 & 646.8 & $17.84 \pm 0.08$ \\
\hline & None & 656.1 & 940.6 & $17.38 \pm 0.05$ \\
\hline & None & 950.2 & 1235.8 & $17.47 \pm 0.06$ \\
\hline & None & 1245.2 & 1530.3 & $17.33 \pm 0.06$ \\
\hline & None & 1539.8 & 1824.1 & $17.31 \pm 0.05$ \\
\hline & None & 1833.4 & 2117.3 & $17.47 \pm 0.06$ \\
\hline & None & 2192.1 & 3066.0 & $17.52 \pm 0.04$ \\
\hline & None & 6228.5 & 7253.4 & $18.07 \pm 0.07$ \\
\hline & None & 7262.9 & 8141.1 & $18.18 \pm 0.07$ \\
\hline & None & 8217.7 & 9091.3 & $18.04 \pm 0.07$ \\
\hline & None & 20706.2 & 21578.7 & $19.43 \pm 0.46$ \\
\hline \multirow[t]{12}{*}{ 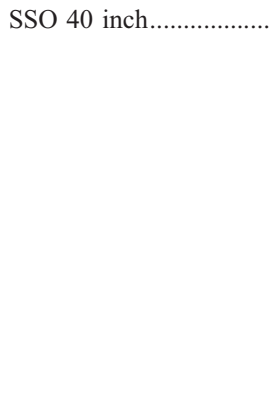 } & None & 7142 & 7242 & $18.38 \pm 0.10$ \\
\hline & $R$ & 7867 & 8167 & $18.63 \pm 0.05$ \\
\hline & $R$ & 8264 & 8564 & $18.63 \pm 0.05$ \\
\hline & $R$ & 8664 & 8964 & $18.77 \pm 0.06$ \\
\hline & $R$ & 9062 & 9362 & $18.70 \pm 0.06$ \\
\hline & $R$ & 9460 & 9760 & $18.84 \pm 0.08$ \\
\hline & $R$ & 16415 & 16715 & $19.63 \pm 0.17$ \\
\hline & $R$ & 16813 & 17113 & $19.55 \pm 0.18$ \\
\hline & $R$ & 17212 & 17512 & $19.79 \pm 0.21$ \\
\hline & $R$ & 17611 & 17911 & $19.59 \pm 0.21$ \\
\hline & $R$ & 18009 & 18309 & $19.64 \pm 0.25$ \\
\hline & $R$ & 81401 & 85346 & $>21.5$ \\
\hline
\end{tabular}

(Henden 2003). Unfortunately, we do not have any color information for the afterglow at the early times. Table 1 shows the results of our photometry for the afterglow, with the light curve plotted in Figure 2.

The ROTSE-IIIa images were bias-subtracted and flatfielded in the standard way. The flat-field image was generated from 30 twilight images. We used SExtractor (Bertin \& Arnouts 1996) to perform the initial object detection and to determine the centroid positions of the stars. We used a robust point-spread function (PSF) fitting code to measure the photometry. We used six bright, well-measured stars from the

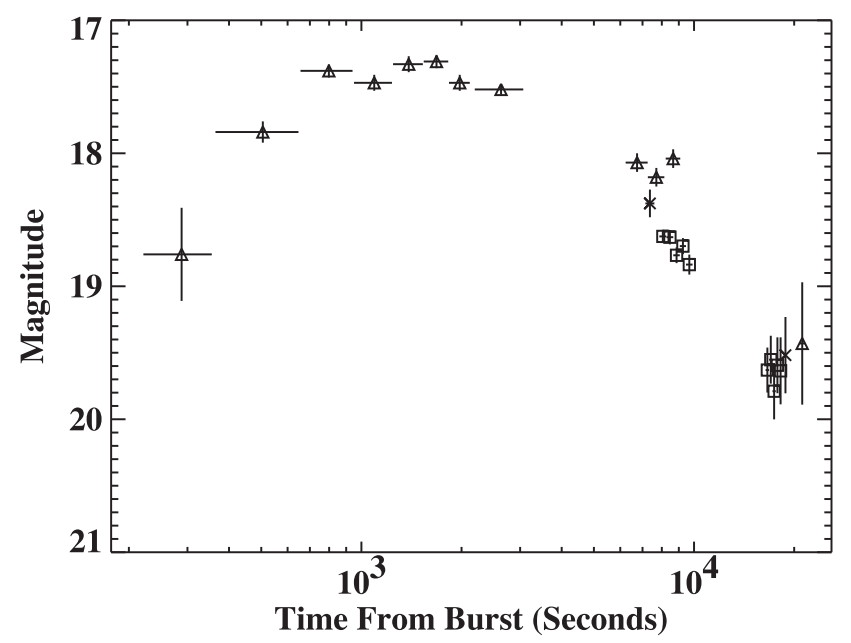

FIG. 2.-Early-time light curve for GRB 030418. The optical emission rises during the first $600 \mathrm{~s}$, slowly varies for $1400 \mathrm{~s}$, and then fades as a power law. The triangles are unfiltered ROTSE-IIIa data, the mult crosses are unfiltered SSO 40 inch data, and the squares are $R$-band SSO 40 inch data.
Henden (2003) list to fit our PSF as well as to determine an $R$-band magnitude zero point. The intrinsic variations in object color generate an rms dispersion of 0.23 between our unfiltered ROTSE magnitudes and the Henden (2003) $R$-band magnitudes.

The SSO 40 inch images were bias-subtracted and flatfielded with a twilight flat in the standard way. We used weighted-aperture photometry and set the magnitude zero point for each frame using between 30 and 90 Henden (2003) stars. The systematic error estimated from comparison with the Henden (2003) stars is about 0.25 mag.

On 2003 July 23, at 06:28:17.45 UT, HETE-2 detected another X-ray-bright GRB (HETE trigger 2777), $23 \mathrm{~s}$ in duration (Prigozhin et al. 2003). The ROTSE-IIIb instrument responded automatically and began taking images within $5 \mathrm{~s}$ of the GCN notice distribution. The first ROTSE exposure began $47 \mathrm{~s}$ after the burst trigger time. The system took $105 \mathrm{~s}$ images, $1020 \mathrm{~s}$ images, and $4060 \mathrm{~s}$ images of the burst field. Much like GRB 030418, the burst counterpart was not found in these early images to limiting magnitudes of 17-18 mag. Later observations by larger telescopes revealed a faint, fading source at $\alpha=21^{\mathrm{h}} 49^{\mathrm{m}} 24^{\mathrm{s}} .40, \delta=-27^{\circ} 42^{\prime} 47^{\prime \prime} .4$ (J2000.0) at 20 mag (Fox et al. 2003a). We co-added sets of 10 images and applied the same PSF fitting technique as described above. The object was not detected in our four earliest co-added images, but the last two images yield marginal detections. We derive $19.5 \pm 0.4$ for the fifth image $(\mathrm{S} / \mathrm{N}=2.7)$ and $19.3 \pm$ 0.4 for the sixth image $(\mathrm{S} / \mathrm{N}=3.1)$. The resulting light curve is shown in Figure 3.

\section{RESULTS}

Figure 2 shows the early-time light curve of GRB 030418 from the ROTSE-IIIa and SSO 40 inch observations. After our first detection, the afterglow brightness is rising. After the rise, 


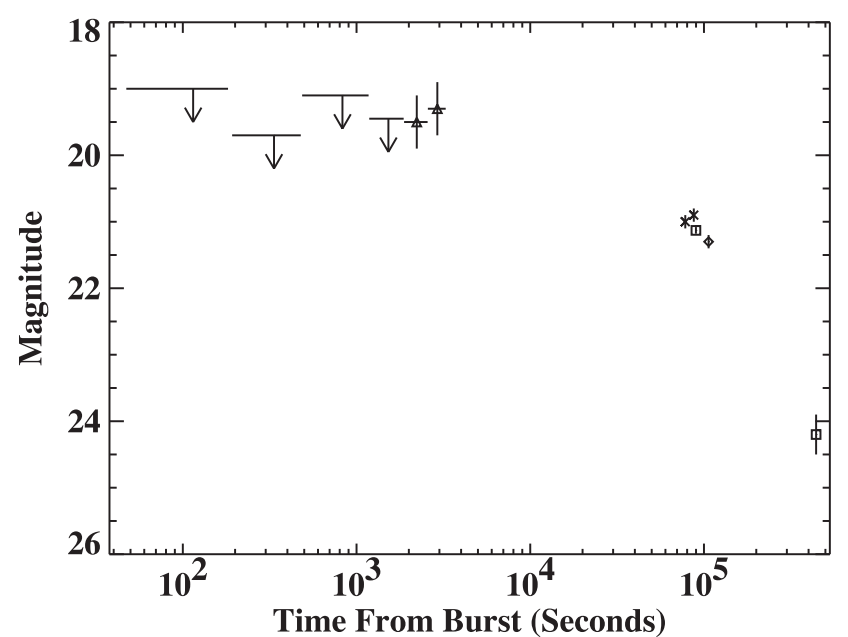

FIG. 3.-Light curve for GRB 030723. The early-time upper limits from ROTSE-IIIb imply behavior similar to GRB 030418. The triangles and upper limits are ROTSE-IIIb data, the mult crosses are Palomar data (Fox et al. 2003a), the squares are Magellan data (Dullighan et al. 2003c, 2003d), and the diamond is Cerro Tololo data (Bond 2003).

the afterglow slowly varies around 17.3 mag for about an hour, before fading following a power law.

Most GRB afterglows have been seen to decline with a sequence of one or more power laws. Two hours after the burst, this is also the case for GRB 030418. Extrapolating this late-time power-law decay back to the early time provokes the question of what happened to the missing optical flux. There are two main alternatives: intrinsically, there were fewer optical photons emitted by the source, or, extrinsically, the optical photons were absorbed after they were created.

The first possibility is not easy to describe without making extensive assumptions about the physics of the shock front or the density of the ambient medium. We have investigated the possibility that the light curve results from the spectral break frequency coming through the optical band (Sari, Piran, \& Narayan 1998). We modeled the flux spectrum as a twocomponent power law as in Sari et al. (1998) for the slow cooling regime, with $F_{\nu} \propto \nu^{1 / 3}$ for $\nu<\nu_{m}$ and $F_{\nu} \propto \nu^{-(p-1) / 2}$ for $\nu>\nu_{m}$, where $\nu_{m}$ is the characteristic synchrotron frequency and $p$ is the spectral index of the electrons, fitted to the late-time power-law decline. The synchrotron frequency decreases as $\nu_{m} \propto t^{-3 / 2}$, with a best-fit value of $\nu_{m}=9.7 \times$ $10^{14} \mathrm{~Hz}$ at $1500 \mathrm{~s}$ after the burst. After integrating the flux in our optical passband, we found that the predicted optical peak is much too sharp and does not reproduce the smooth rollover we see in the light curve of GRB 030418, as can be seen as the dotted line in Figure 4. This fast transition is not caused by the sharpness of the frequency break but rather by the rapidity with which the break moves through the optical band.

We next investigated the possibility that we are seeing the transition from fast cooling to slow cooling, as in Sari et al. (1998). In addition to our model described above, we added the cooling frequency, $\nu_{c}$, which decays as $\nu_{c} \propto t^{-1 / 2}$. By fitting the initial values of $\nu_{c}$ and $\nu_{m}$, the modeled light curve is plotted as the dashed line in Figure 4. This model succeeds in describing the flat peak of the light curve but does not describe the rise at early times. In addition, the best-fit frequency values at $1500 \mathrm{~s}$ for $\nu_{c}=4.6 \times 10^{14} \mathrm{~Hz}$ and $\nu_{m}=1.8 \times 10^{15} \mathrm{~Hz}$ are not physically reasonable, according to the prescription in Granot \& Sari (2002).
Recently, evidence has emerged linking GRBs to corecollapse supernovae of massive stars, including the detection of a supernova spectrum in the afterglow of GRB 030329 (Stanek et al. 2003; Hjorth et al. 2003). A key consequence of a massive star progenitor is that the GRB occurs inside a massive stellar wind (Mészáros, Rees, \& Wijers 1998; Chevalier \& Li 1999). To date, most of the literature has focused on the generation of the shock front in a wind medium without consideration of other physical effects. Here, we investigate the possibility that the absorption of the optical photons by this circumburst environment can explain the early behavior of the GRB 030418 light curve. Although a prompt optical/UV flash might sublimate the dust (Waxman \& Draine 2000), if this were the entire story then we would likely have seen the afterglow decay at the early time.

The stellar wind density profile is $\rho=\rho_{0} r_{0}^{2} / r^{2}$, assuming a constant mass-loss rate from the massive star. The optical depth scale then becomes $\tau=\beta_{r} / r$, and the optical flux absorbed by the circumburst medium is attenuated as $e^{-\beta_{r} / r}$. If we assume that at the early time the emitting shell is moving at a roughly constant velocity with a bulk Lorentz factor of $\Gamma$, then the distance traveled is related to the time in the Earth's frame of reference as $r \simeq \frac{1}{2} \Gamma^{2} c t$. Therefore, the attenuation as a function of time goes as $e^{-\beta_{t} / t}$. As the standard model has the afterglow fading as a power law after the initial energy injection into the circumburst medium, we assume that the power-law decline applies at all relevant times. We can then fit the rise and fall of the afterglow with an attenuated power-law function,

$$
F_{\nu}=F_{0} t^{-\alpha} e^{-\beta_{t} / t}
$$

We derive the three free parameters of this fitting function, $F_{0}, \alpha$, and $\beta_{t}$, empirically via a linear regression fit to the observed afterglow light curve. The best-fit function is plotted in Figure 4, with $\alpha=1.36 \pm 0.02$ and $\beta_{t}=1.81( \pm 0.05) \times$ $10^{3} \mathrm{~s}$, and $F_{0}$ is an arbitrary normalization factor. The formal errors quoted are from the linear regression fit. The fit has a $\chi^{2}$

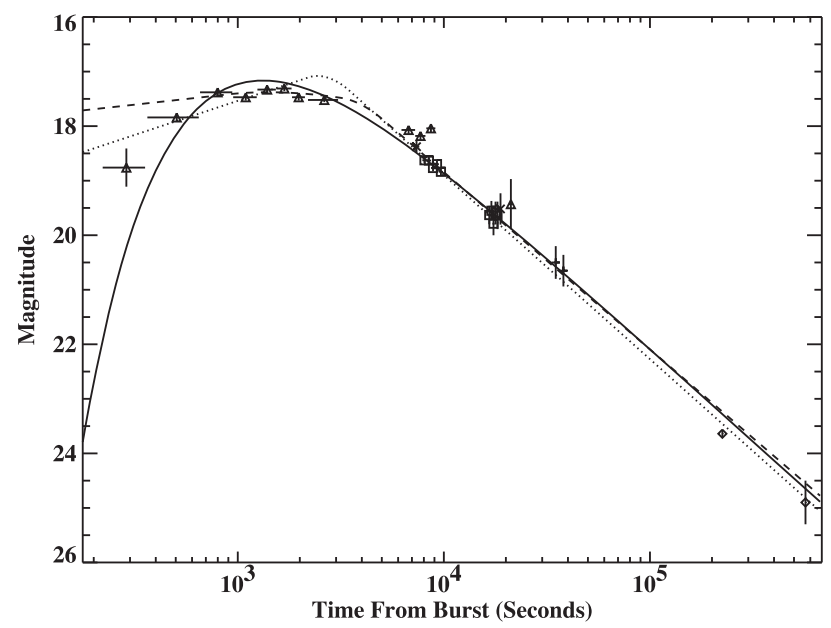

FIG. 4.- - Light curve for GRB 030418 with the best-fit model superposed. The solid line fit is of the form $F_{\nu}=F_{0} t^{-\alpha} e^{-\beta_{t} / t}$, where $\alpha=1.36 \pm 0.02$. The dotted line fit is a model with slow cooling as in Sari et al. (1998), while the dashed line is a model with both slow and fast cooling. The triangles are ROTSE-IIIa observations, the mult crosses and squares are SSO 40 inch observations, the plus signs are Loiano telescope data from Ferrero et al. (2003), and the diamonds are Magellan telescope data from Dullighan et al. (2003a, 2003b). 
per degree of freedom of 8.7. This large value is mostly due to local effects in the data at $8000 \mathrm{~s}$.

From Figure 4, it can be seen that this physical model is able to represent the gross features of the light curve. We will now show that the observed value for the attenuation timescale, $\beta_{t}$, is consistent with reasonable assumptions about the circumburst medium. To estimate the mass loss implied by $\beta_{t}$, we assume that the primary absorbing medium consists of dust grains similar in composition to the interstellar medium (ISM). The opacity of the ISM can be approximately modeled as $\kappa_{\nu}=\left(5 \times 10^{-13} \mathrm{~cm}^{2} \mathrm{~g}^{-1} \mathrm{~s}\right) \nu$ (Draine \& Lee 1984; Adams \& Shu 1985). Although there is no redshift measurement for this burst, we assume a typical redshift of $z \sim 1$, which puts the peak response of our CCD at $\lambda \sim 325 \mathrm{~nm}$ in the GRB local frame of reference.

We estimate the wind velocity as $v_{\text {wind }} \sim 100 \mathrm{~km} \mathrm{~s}^{-1}$, the escape velocity from a $50 M_{\odot}$ star with a radius $2000 R_{\odot}$, a typical value for a red supergiant. Finally, we assume that the bulk Lorentz factor of the GRB ejecta is 100 . The derived mass-loss rate for the progenitor star is then

$$
\begin{aligned}
\frac{d M}{d t}= & 1.2 \times 10^{-3} \frac{M_{\odot}}{\mathrm{yr}}\left(\frac{\Gamma}{100}\right)^{2} \frac{\beta_{t}}{1800} \frac{450 \mathrm{~cm}^{2} \mathrm{~g}^{-1}}{\kappa} \\
& \times \frac{v}{100 \mathrm{~km} \mathrm{~s}^{-1}}
\end{aligned}
$$

where $\Gamma$ is the bulk Lorentz factor, $\kappa$ is the opacity, and $v$ is the velocity of the stellar wind. With our assumptions, a mass-loss rate of $1.2 \times 10^{-3} M_{\odot} \mathrm{yr}^{-1}$ is high compared with that of late-stage, high-mass stars, which is typically $10^{-5}$ to $10^{-4} M_{\odot} \mathrm{yr}^{-1}$ (Garcia-Segura, Langer, \& Mac Low 1996a; Garcia-Segura, Mac Low, \& Langer 1996b). However, such a mass-loss rate may not be out of line for the extreme stellar masses required by the prevailing collapsar/hypernova models of GRB progenitors. This estimate is also dependent on the accuracy of our assumed value for the bulk Lorentz factora factor of 3 lower would reduce the mass loss by a factor of 10. Additionally, our estimate is only reasonable with a massloss rate typical of a red supergiant, not a Wolf-Rayet star as discussed as a possible progenitor for GRB 021004 (Schaefer et al. 2003).

\section{DISCUSSION}

GRB 030418 is one of the earliest afterglows yet imaged, with the initial detection only $76 \mathrm{~s}$ after the cessation of the gamma-ray activity. Unlike GRB 990123, this burst does not appear to have a prompt optical counterpart that can be attributed to the reverse shock. However, if our model of local extinction is correct, we would not expect to see any prompt emission; this model implies optical extinction of roughly $20 \mathrm{mag}$ at $100 \mathrm{~s}$, near the time gamma-ray emission ceased.

A backward extrapolation of the late power-law decline overestimates the optical emission from GRB 030418. Our model of local dust absorption in a stellar wind medium is a useful way of characterizing the data. Unlike frequency-break models, our model is able to describe the steep rise and slow rollover of the light curve. This early-time behavior is far from universal, as several bursts, including GRB 990123, and GRB 021211, had more emission than predicted from the late power-law decline. However, we already have evidence that the light-curve behavior of GRB 030418 is not unique. Our early-time observations of GRB 030723 show a similarity to the light curve of GRB 020418. Given the observational biases against detecting such dim fading objects, it is not surprising that this class of GRB afterglows is just now being discovered as a consequence of more accurate coordinate determinations in space and more sensitive optical detectors on the ground.

One of the main consequences of our absorption model in a stellar wind medium is that some afterglows will rise very steeply in the early time. It is at this very early time that the degeneracy between our model and the frequency-break models is broken. Another consequence of a dusty local environment is that the extinction in the optical bands will be much greater than in the near-infrared. Prompt multicolor observations will therefore be invaluable so as to firmly establish whether or not this type of initial behavior is due to optical absorption as described above.

This work has been supported by NASA grants NAG55281 and F006794, NSF grants AST 01-19685 and 01-05221, the Australian Research Council, the University of New South Wales, and the University of Michigan. Work performed at LANL is supported by NASA SR\&T through Department of Energy (DOE) contract W-7405-ENG-36 and through internal LDRD funding. Special thanks to the staff at Siding Spring and McDonald Observatories, to Scott Barthlemy for maintaining the GCN, and to Fred Adams for his illuminating discussion of dust absorption.
Adams, F. C., \& Shu, F. H. 1985, ApJ, 296, 655

Akerlof, C., et al. 1999, Nature, 398, 400

2000, ApJ, 532, L25

2003, PASP, 115, 132

Bertin, E., \& Arnouts, S. 1996, A\&AS, 117, 393

Bond, H. E. 2003, GCN Circ. 2329 (http://gcn.gsfc.nasa.gov/gen/gen3/2329.gcn3)

Chevalier, R. A., \& Li, Z. 1999, ApJ, 520, L29

Draine, B. T., \& Lee, H. M. 1984, ApJ, 285, 89

Dullighan, A., Butler, N. R., Ricker, G. R., Elliot, J. L., Clancy, K., \& Osip, D. 2003a, GCN Circ. 2168 (http://gcn.gsfc.nasa.gov/gcn/gcn3/2168.gcn3) 2003b, GCN Circ. 2236 (http://gcn.gsfc.nasa.gov/gcn/gcn3/2236.gcn3)

Dullighan, A., Butler, N., Vanderspek, R., Villasenor, J., \& Ricker, G. 2003c, GCN Circ. 2326 (http://gcn.gsfc.nasa.gov/gen/gcn3/2326.gcn3) 2003d, GCN Circ. 2336 (http://gcn.gsfc.nasa.gov/gcn/gcn3/2336.gcn3)

Ferrero, P., Pizzichini, G., Bartolini, C., Guarnieri, A., Piccioni, A., \& de Blasi, A. 2003, GCN Circ. 2284 (http://gcn.gsfc.nasa.gov/gcn/gcn3/ 2284.gcn3)

\section{REFERENCES}

Fox, D. B., Kaplan, D. L., Cenko, B., Kulkarni, S. R., \& Nechita, W. A. 2003a, GCN Circ. 2323 (http://gcn.gsfc.nasa.gov/gen/gcn3/2323.gcn3)

Fox, D. W., et al. 2003b, ApJ, 586, L5 2003c, Nature, 422, 284

Garcia-Segura, G., Langer, N., \& Mac Low, M.-M. 1996a, A\&A, 316, 133

Garcia-Segura, G., Mac Low, M.-M., \& Langer, N. 1996b, A\&A, 305, 229

Granot, J., \& Sari, R. 2002, ApJ, 568, 820

Henden, A. 2003, GCN Circ. 2216 (http://gcn.gsfc.nasa.gov/gcn/gcn3/ 2216.gcn3)

Hjorth, J., et al. 2003, Nature, 423, 847

Kehoe, R., et al. 2001, ApJ, 554, L159

Klose, S., et al. 2003, ApJ, 592, 1025

Li, W., Filippenko, A. V., Chornock, R., \& Jha, S. 2003, ApJ, 586, L9

Mészáros, P., Rees, M. J., \& Wijers, R. A. M. J. 1998, ApJ, 499, 301

Park, H. S., et al. 1999, A\&AS, 138, 577

Price, P. A., McNaught, R., Garradd, G. J., Verrender, M. A., \& Smith, T. M. 2003, GCN Circ. 2148 (http://gcn.gsfc.nasa.gov/gcn/gcn3/2148.gcn3) 
Prigozhin, G., et al. 2003, GCN Circ. 2313 (http://gcn.gsfc.nasa.gov/gcn/gen3/ 2313.gen3)

Sari, R., Piran, T., \& Narayan, R. 1998, ApJ, 497, L17

Schaefer, B. E., et al. 2003, ApJ, 588, 387
Shirasaki, Y., et al. 2003, GCN Circ. 2210 (http://gen.gsfc.nasa.gov/gen/gen3/ 2210.gcn3)

Stanek, K. Z., et al. 2003, ApJ, 591, L17

Waxman, E., \& Draine, B. T. 2000, ApJ, 537, 796 